\title{
Retraction: Synthesizing Neural Networks and Randomized Algorithms
}

\author{
Yonghong Shao ${ }^{1}$, Qingyue Kong ${ }^{2}$, and Yingying $\mathrm{Ma}^{3}$ \\ ${ }^{1}$ Department of Art and Design, Yiwu Industrial and Commercial College, \\ Yiwu, China \\ ${ }^{2}$ Department of Information Engineering, Hebei Chemial \& Pharmaceutical College, \\ Shijiazhuang, China \\ ${ }^{3}$ College of Business Administration, South China University of Technology, \\ Guangzhou, China \\ shaoyonghong3046@163.com, Kongqingyue@163.com, \\ mayingying@163.com
}

Several conference proceedings have been infiltrated by fake submissions generated by the SCIgen computer program. Due to the fictional content the chapter "Synthesizing Neural Networks and Randomized Algorithms" by "Yonghong Shao, Qingyue Kong, and Yingying Ma" has been retracted by the publisher. Measures are being taken to avoid similar breaches in the future. 